\title{
Testing Gravity Theory With Extreme Mass-Ratio Inspirals: Recent Progress ${ }^{\dagger}$
}

\author{
Shu-Cheng Yang ${ }^{1,2}$, Shuo Xin ${ }^{1,3}$ and Chen Zhang ${ }^{1,2}$ and Wen-Biao Han ${ }^{1,2, *}$ \\ 1 Shanghai Astronomical Observatory, CAS, Shanghai 200030, China; ysc@shao.ac.cn (S.-C.Y.); \\ xinshuo@tongji.edu.cn (S.X.); zc@shao.ac.cn (C.Z.) \\ 2 School of Astronomy and Space Science, University of Chinese Academy of Sciences, Beijing 100049, China \\ 3 School of Physics Sciences and Engineering, Tongji University, Shanghai 200092, China \\ * Correspondence: wbhan@shao.ac.cn \\ + Presented at the meeting Recent Progress in Relativistic Astrophysics, Shanghai, China, 6-8 May 2019.
}

Published: 14 October 2019

check for updates

\begin{abstract}
A compact object captured by a supermassive black hole, named as extreme-mass-ratio inspiral (EMRI), is one of the most important gravitational wave sources for low-frequency interferometers such as LISA, Taiji, and TianQin. EMRIs can be used to accurately map the space-time of the central massive body. In the present paper, we introduce our recent progress on testing gravity theory with EMRIs. We demonstrate how to constrain gravitational wave dispersion and measure the deviation of the central body from the Kerr black hole. By using binary-EMRIs, the gravitational recoil and mass loss due to merger will be measured in a higher accuracy compared with the current LIGO observations. All these potential constrains and measurements will be useful for test of the gravity theory.
\end{abstract}

Keywords: gravity theory testing; extreme-mass-ratio inspirals; gravitational waves

\section{Introduction}

Compact binary inspirals, which are composed by orbiting pairs of compact objects, are important gravitational wave(GW) sources. Nowadays, the GWs of comparable mass-ratio binary coalescences, such as binary black hole (BBH) and binary neutron star (BNS) have been detected by advanced LIGO (aLIGO) and advanced Virgo [1,2]. However, most large mass ratio cases, such as extreme-mass-ratio inspirals (EMRIs) and intermediate-mass-ratio inspirals (IMRIs) [3], whose GWs belong to low-frequency GW band, could only be detected by space-borne interferometers such as Laser Interferometer Space Antenna (LISA) [4], Taiji [5], and TianQin [6]. EMRI systems are produced when a massive black hole (MBH) captures small compact objects, which could be stellar-mass black holes (SMBHs), neutron stars, and white dwarfs. Furthermore, BBHs could also be captured by an $\mathrm{MBH}$, and after that a binary-EMRI system (b-EMRI) is produced [7,8]. Unlike comparable binaries, the GW signals of typical EMRI systems could last for years in the space-borne GW detectors' band, which could give us ample information about the space-time geometry at the immediate exterior of the MBH $[9,10]$. Meanwhile, the signal to noise ratio(SNR) of EMRI's GW signals could be better than BBH. All of these make EMRI an accurate tool for GW astronomy, astrophysics, cosmology, and fundamental physics tests.

In this paper, we introduce our recent progress on testing gravity theory with EMRIs, and this paper is organized as follows. Sections 2-4 introduce our recent works. The research in Section 2 tests the Kerr metric hypothesis of BHs with EMRIs. The research in Section 3 gives a bound on the mass of graviton and tests the dispersion effect of GWs. Section 4 talks about the b-EMRIs. We found that 
b-EMRIs could not only accurately measure mass loss and recoil velocity of the binary merger, but also confirm or rule out GW dispersion. Finally, the conclusion is given in the last section.

\section{Testing the Kerr black hole hypothesis with EMRIs}

Testing general relativity (GR) and Kerr metric in a strong field regime is an important goal of future space detectors such as LISA [11]. However, as is shown in [12], large parameter space EMRI has a "confusion problem" that could prevent us from testing alternative gravity theory or metric. In [13], by matching EMRI signals, we investigate the possibility of testing the Kerr hypothesis against a parameterized non-Kerr metric (we refer to this metric [14] as KRZ metric).

Within the time scale before radiation flux, which plays an important role, we find that EMRIs from either equatorial orbits or inclined orbits suffer from the "confusion problem". Figure 1 shows the upper and lower bounds of the deformation parameters with respect to $\mathrm{BH}$ spin for equatorial orbit and inclined orbit. As is shown in Figure 1, small and moderate deviations from the Kerr spacetime $\left(\left|\delta_{i}\right|<1\right)$ can be discerned only when spin parameter is extreme. However, as the radiation flux became significant in orbit evolution, the waveforms related with a non-Kerr metric can not be mimicked by the waveform templates produced from Kerr black hole, please see [13] for details.
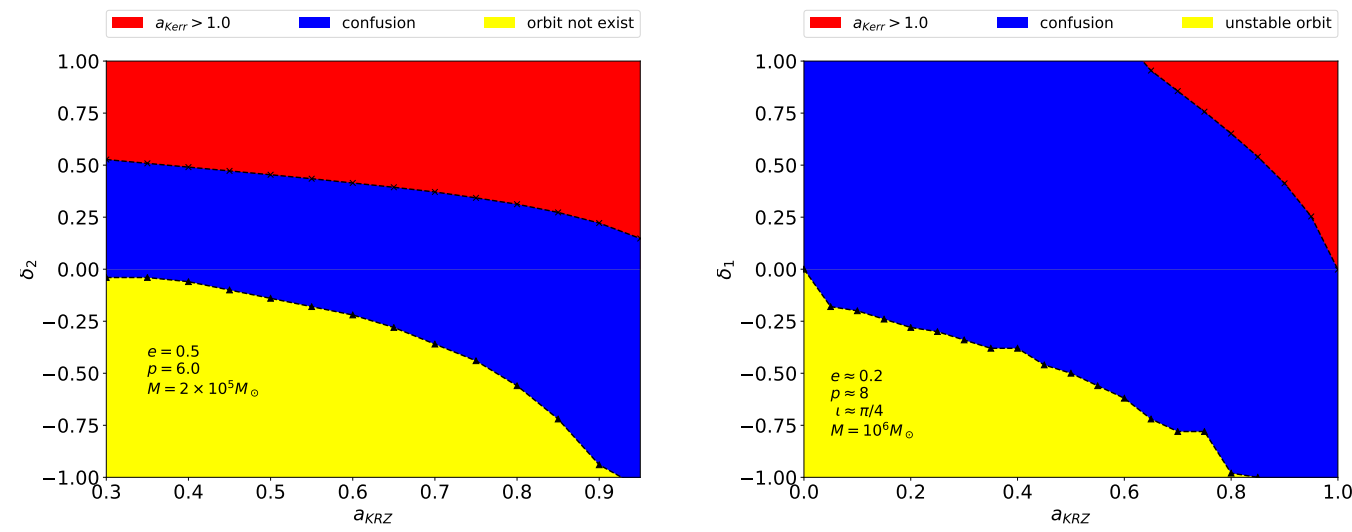

Figure 1. Upper and lower limits of $\delta_{i}$, whose signals can be confused with Kerr waveform, on the $\delta_{i}-a_{\text {KRZ }}$ parameter plane, set by equatorial orbit requiring $a_{\text {Kerr }}<1$ and stable orbit. Other parameters are shown on the figure. Waveforms in the red parameter region, when trying to equate orbital frequencies by varying $(M, a)$, have results in $a_{\text {Kerr }}$ larger than 1 . Waveforms in the blue region can be confused with Kerr waveform. Orbits in the yellow region do not exist. Left panel: for equatorial orbit. Right panel: for inclined orbit.

\section{Testing GW Dispersion}

Some modified gravity theories predict dispersion phenomena in the propagation of gravitational waves [15-18]. In a massive graviton background, we have the dispersion relation $[19,20]$

$$
\frac{v_{\mathrm{g}}^{2}}{c^{2}}=1-\frac{m_{\mathrm{g}}^{2} c^{4}}{E^{2}}
$$

where $v_{\mathrm{g}}$ denotes the speed of the graviton, $E$ denotes the total energy of the graviton, and $m_{\mathrm{g}}$ denotes the rest mass of graviton. The Compton wavelength of the graviton: $\lambda_{\mathrm{g}}=h / m_{\mathrm{g}} \mathrm{c}$. In GR, we have $m_{\mathrm{g}}=0$ and $\lambda_{\mathrm{g}} \rightarrow \infty$.

In [21], we demonstrate that this dispersion will induce an observable deviation of waveforms if the orbits have large eccentricities, which could give a more stringent constraint on $m_{\mathrm{g}}$ and $\lambda_{\mathrm{g}}$. The mechanism is that the waveform modes with different frequencies will be emitted at the same time due to the existence of eccentricity. In this situation, considering two gravitons emitted at $t_{\mathrm{e}}$ and 
$t_{\mathrm{e}}^{\prime}$ with different frequencies of $f_{\mathrm{e}}$ and $f_{\mathrm{e}}^{\prime}$, which will be received at corresponding arrival times $t_{\mathrm{a}}$ and $t_{a}^{\prime}$, the delay of arrival times of two gravitons could be expressed as $[19,20]$

$$
\Delta t_{\mathrm{a}}=(1+Z) \frac{c D}{2 \lambda_{\mathrm{g}}^{2}}\left(\frac{1}{f_{\mathrm{e}}^{2}}-\frac{1}{f_{\mathrm{e}}^{\prime 2}}\right)
$$

where $Z$ is the cosmological redshift, and $D$ and luminosity distance $D_{L}$ could be written as [20]

$$
\begin{gathered}
D=\frac{c(1+Z)}{H_{0}} \int_{0}^{Z} \frac{\left(1+z^{\prime}\right)^{-2} d z^{\prime}}{\sqrt{\Omega_{\mathrm{M}}\left(1+z^{\prime}\right)^{3}+\Omega_{\Lambda}}}, \\
D_{L}=\frac{c(1+Z)}{H_{0}} \int_{0}^{Z} \frac{d z^{\prime}}{\sqrt{\Omega_{\mathrm{M}}\left(1+z^{\prime}\right)^{3}+\Omega_{\Lambda}}},
\end{gathered}
$$

where $H_{0}, \Omega_{\mathrm{M}}$, and $\Omega_{\Lambda}$ the denote Hubble constant, matter density parameter today, and dark energy density parameter today, respectively. The radiation density parameter today, $\Omega_{R}$, is omitted here. As is shown in Figure 2, during the propagation of such GW, because of the dispersion, the arrival time of different modes will be different and will then produce the deviation and dephasing of waveforms compared with GR. Note, in this work, we did not consider other gravity effects such as gravitational lens, which could be used to test gravity theory as well [22]. Further consideration on other gravity effects may lead to interesting conclusions on the field of gravity theories testing.

This kind of dispersion phenomena related with EMRI could be observed by space-borne detectors and obtains an improved constraint on $m_{\mathrm{g}}\left(\right.$ or $\lambda_{\mathrm{g}}$ ). Figures 3 and 4 show the matches (maximized overlap) between dispersive and nondispersive GWs. We set a criteria value of 0.97 on matches, by which we could get a constraint. As shown in Figure 3, the constraint on graviton mass could be improved approximately three orders of magnitude compared to the current level of $m_{\mathrm{g}} \leq 7.7 \times$ $10^{-23} \mathrm{eV} / \mathrm{c}^{2}\left(\lambda_{\mathrm{g}}>1.6 \times 10^{13} \mathrm{~km}\right)$ [23]. Moreover, as shown in Figure 4, we find that the dispersion effect may also be constrained better than the current result by ground-borne detectors if a highly eccentric intermediate-mass-ratio inspirals be observed.
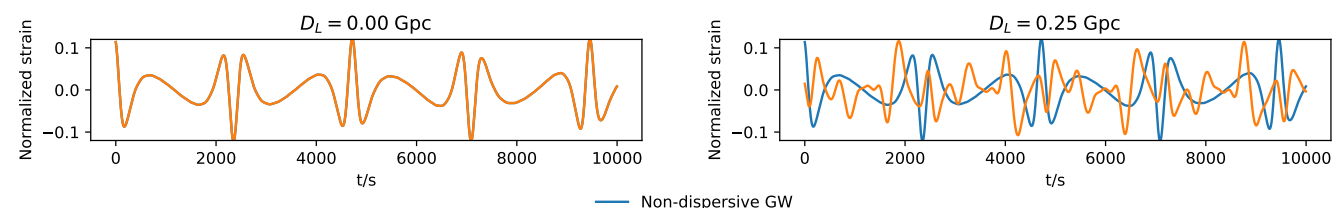

- Non-dispersive GW
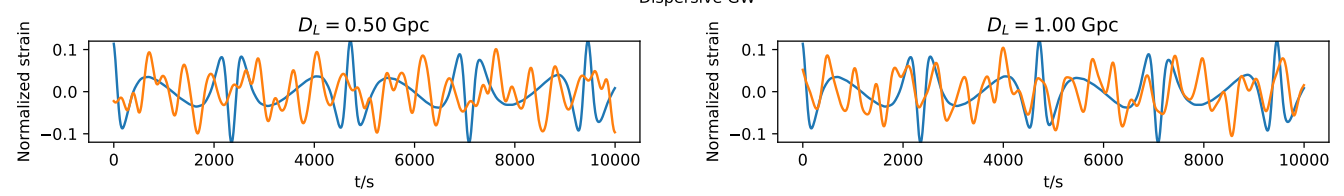

Figure 2. The distortion of gravitational waves from an extreme-mass-ratio inspiral during propagation (EMRI, $\lambda_{\mathrm{g}}=1.6 \times 10^{13} \mathrm{~km}$ ). Total mass: $M=10^{6} M_{\odot}$; symmetric mass ratio: $v=10^{-5}, e=0.5$, $p=12 M$, and $a=0.9$. We set the luminosity distance of sources $D_{L}$ from 0 to $1.00 \mathrm{Gpc}$. The orange curves represent the waveforms with dispersion during the propagation, and the cyan one is the nondispersive ones. 


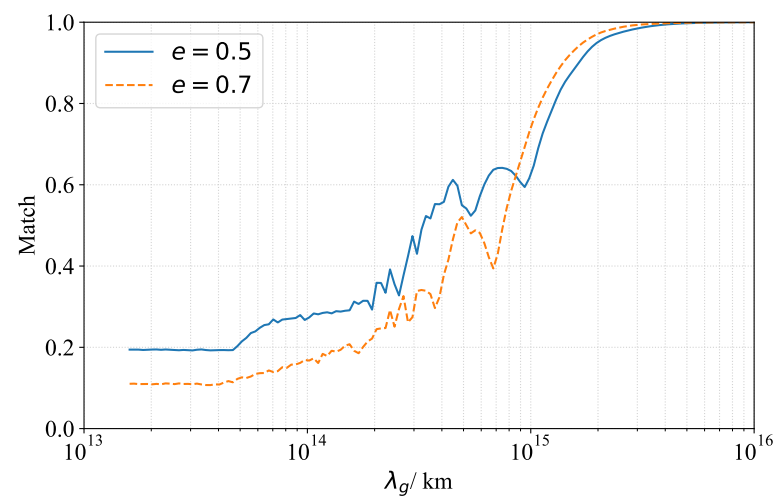

Figure 3. Matches between dispersive and nondispersive GW series in different $e$ and $\lambda_{\mathrm{g}}$ (LISA band). The curves begins at $\lambda_{g}=1.6 \times 10^{13} \mathrm{~km}$, the total masses of systems are $M=10^{6} M_{\odot}$, where the symmetric mass ratio $v=10^{-5}, e=0.5$ (cyan line) and $e=0.7$ (orange line), $p=12 \mathrm{M}$, and $a=0.9$. For the GW series, the duration is $2^{25} \mathrm{~s}(\sim 1.06$ year) and the sampling frequency is $0.25 \mathrm{~Hz}$. We set $D_{L}=1.00 \mathrm{Gpc}$, where $Z \approx 0.20$ and $D \approx 0.83 \mathrm{Gpc}$.

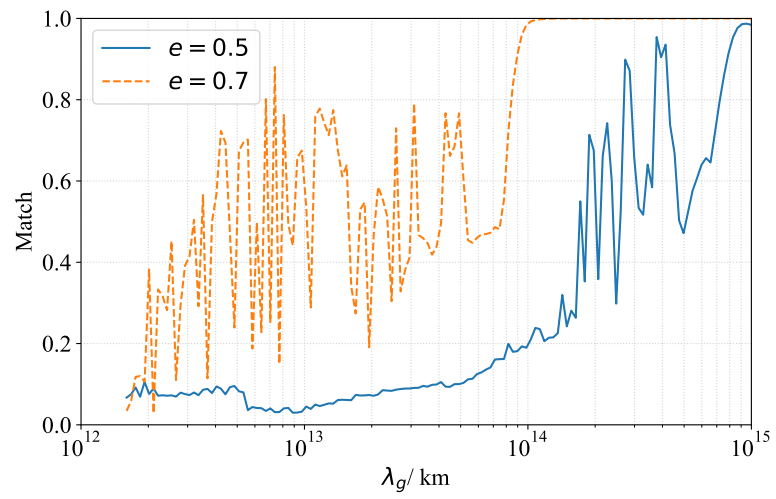

Figure 4. Matches between dispersive and nondispersive GW series in different $e$ and $\lambda_{\mathrm{g}}$ (aLIGO band). The curves begin at $\lambda_{g}=1.6 \times 10^{12} \mathrm{~km}$, the total masses of the systems are $M=500 M_{\odot}$, where the symmetric mass ratio $v=10^{-2}, e=0.5$ (cyan line) and $e=0.7$ (orange line), $p=12 \mathrm{M}$, and $a=0.95$. For the GW series, the duration is $2^{7} \mathrm{~s}(128 \mathrm{~s})$ and the sampling frequency is $2^{14} \mathrm{~Hz}$. We set $D_{L}=1.00 \mathrm{Gpc}$, where $Z \approx 0.20$ and $D \approx 0.83 \mathrm{Gpc}$.

\section{New Multiband GW Source: b-EMRI}

BBHs could also be captured by a MBH, and after that a b-EMRI is produced [7,8]. In [8], we carefully investigate the formation of b-EMRIs; Figure 5 shows three evolutionary stages of a b-EMRI. We also find that $~ 30 \%$ of the captured binaries coalesce due to the perturbation by the massive bodies, resulting in a merger rate of $0.03 \mathrm{Gpc}^{-3} \mathrm{yr}^{-1}$ in the most optimistic scenario. High-frequency $\left(10^{2} \mathrm{~Hz}\right) \mathrm{GWs}$ detectable by ground-based observatories could also be generated by the coalescence, making these b-EMRIs ideal targets for future multi-band GW observations.

In [24], we further show that the coalescence of BBHs in b-EMRIs could be detected by LISA as glitches in EMRIs signals to test gravity theories. Our simulations suggest that the experiment could measure the mass and linear momentum lost via GW radiation, as well as constrain the mass of gravitons to a precision that is one order of magnitude better than the current limit. Meanwhile, we could also accurately measure the recoil velocity of binary merger and linear momentum in b-EMRIs, as shown in Figure 6. 


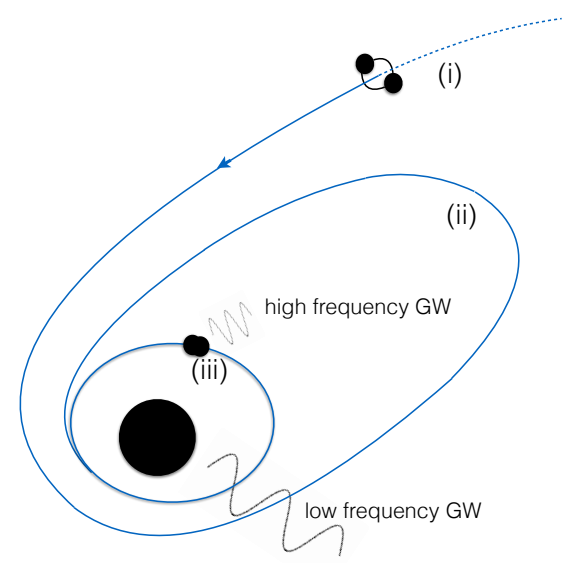

Figure 5. Three evolutionary stages of a binary extreme-mass-ratio inspiral (b-EMRI). First, (i) a compact black hole binary (BHB) is captured to a bound orbit around a supermassive black hole ( $\mathrm{SMBH}$ ) because the pericenter distance becomes comparable to the tidal-disruption radius, $R_{\mathrm{t}}$. Next, (ii) the outer binary circularizes due to gravitational wave (GW) radiation, and the GW frequency lies in the band of a space-borne GW detector. Finally, (iii) the tidal force of the SMBH becomes strong enough to excite the eccentricity of the inner BHB and drive it to merge. The merger produces high-frequency GWs.

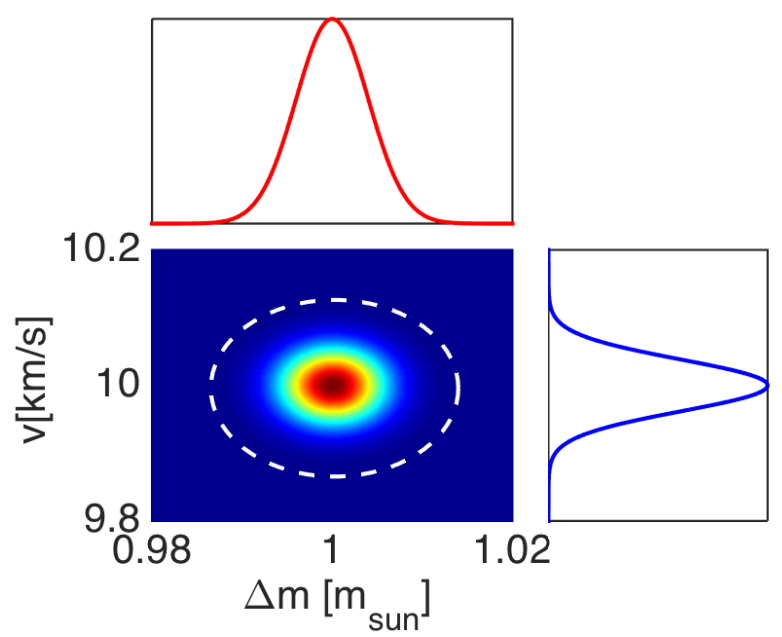

Figure 6. Likelihood of the recoil velocity $v$ and mass loss $\Delta m$ derived from the Fisher matrix. The white dashed ellipse shows the $3 \sigma$ confidence level. The upper and right-hand panels show the marginalized probability distribution for $\Delta m$ and $v$, respectively.

\section{Discussion}

To conclude, in the present paper, we introduce our recent progress on testing gravity theory with EMRIs, including the Kerr metric hypothesis, the dispersion of GW, and testing gravity theory with b-EMRIs. We demonstrate the great potential of EMRI signals on the test of gravity theory, which will be an important scientific target for space-based detectors. Meanwhile, with efforts on the future space-based detectors, there will be some challenges and chances. On the one hand, accurate templates with long evolution are necessary. On the other hand, during one-year orbital evolution, unsteady and non-Gaussian noises from both the GW source and detector could happen and produce uncertainty on the parameter estimation. Therefore, people in this realm still have many opportunities to take. 
Author Contributions: conceptualization, Wen-Biao Han; methodology, Shu-Cheng Yang, Shuo Xin, Chen Zhang and Wen-Biao Han; software, Shu-Cheng Yang, Shuo Xin and Wen-Biao Han; validation, Wen-Biao Han; formal analysis, Shu-Cheng Yang, Shuo Xin and Wen-Biao Han; investigation, Shu-Cheng Yang, Shuo Xin and Wen-Biao Han; resources, Wen-Biao Han; data curation, Shu-Cheng Yang, Shuo Xin and Wen-Biao Han; writing-original draft preparation, Shu-Cheng Yang; writing-review and editing, Wen-Biao Han and Shu-Cheng Yang; visualization, Wen-Biao Han, Shu-Cheng Yang and Shuo Xin; supervision, Wen-Biao Han; project administration, Wen-Biao Han; funding acquisition, Wen-Biao Han.

Funding: This work is supported by NSFC No. 11773059.

Conflicts of Interest: The authors declare no conflicts of interest.

\section{Abbreviations}

The following abbreviations are used in this manuscript:

$\begin{array}{ll}\text { GW } & \text { gravitational wave } \\ \text { BBH } & \text { binary black hole } \\ \text { BNS } & \text { binary neutron star } \\ \text { EMRI } & \text { extreme-mass-ratio inspiral } \\ \text { IMRI } & \text { intermediate-mass-ratio inspiral } \\ \text { aLIGO } & \text { Advanced Laser Interferometer Gravitational-Wave Observatory } \\ \text { LISA } & \text { Laser Interferometer Space Antenna } \\ \text { MBH } & \text { massive black hole } \\ \text { SMBH } & \text { stellar-mass black holes } \\ \text { b-EMRI } & \text { binary-EMRI } \\ \text { SNR } & \text { signal-to-noise ratio } \\ \text { GR } & \text { general relativity }\end{array}$

\section{References}

1. Abbott, B.P.; Abbott, R.; Abbott, T.D.; Abernathy, M.R.; Acernese, F.; Ackley, K.; Adams, C.; Adams, T.; Addesso, P.; Adhikari, R.X.; et al. Observation of Gravitational Waves from a Binary Black Hole Merger. Phys. Rev. Lett. 2016, 116, 688-16.

2. Abbott, B.P. GW170817: Observation of Gravitational Waves from a Binary Neutron Star Inspiral. Phys. Rev. Lett. 2017, 119, 61-18.

3. Amaro-Seoane, P.; Gair, J.R.; Freitag, M.; Miller, M.C.; Mandel, I.; Cutler, C.J.; Babak, S. Intermediate and extreme-mass-ratio inspirals-astrophysics, science applications and detection using LISA. Class. Quantum Grav. 2007, 24, R113-R169.

4. Danzmann, K.; LISA Study Team. LISA: Laser interferometer space antenna for gravitational wave measurements.Class. Quantum Grav. 1996, 13, A247-A250.

5. Hu, W.-R.; Wu, Y.-L. The Taiji Program in Space for gravitational wave physics and the nature of gravity. Natl. Sci. Rev. 2017, 4, 685-686.

6. Luo, J.; Chen, L.S.; Duan, H.Z.; Gong, Y.G.; Hu, S.; Ji, J.; Liu, Q.; Mei, J.; Milyukov, V.; Sazhin, M.; et al. TianQin: A space-borne gravitational wave detector. Class. Quantum Grav. 2016, 33, 035010.

7. Addison, E.; Gracia-Linares, M.; Laguna, P.; Larson, S.L. Busting up binaries: Encounters between compact binaries and a supermassive black hole. Gen Relat. Grav. 2019, 51, 061102.

8. Chen, X.; Han, W.-B. Extreme-mass-ratio inspirals produced by tidal capture of binary black holes. Commun. Phys. 2018, 1, 53.

9. Gair, J.R.; Vallisneri, M.; Larson, S.L.; Baker, J.G. Testing General Relativity with Low-Frequency, Space-Based Gravitational-Wave Detectors. Living Rev. Relat. 2013, 16, 173001.

10. Barausse, E.; Cardoso, V.; Pani, P. Can environmental effects spoil precision gravitational-wave astrophysics? Phys. Rev. D 2014, 89, 379.

11. Scharre, P.D.; Will, C.M. Testing scalar-tensor gravity using space gravitational-wave interferometers. Phys. Rev. D 2002, 65, 38.

12. Barausse, E.; Rezzolla, L.; Petroff, D.; Ansorg, M. Gravitational waves from extreme mass ratio inspirals in nonpure Kerr spacetimes. Phys. Rev. D 2007, 75, 581. 
13. Xin, S.; Han, W.-B.; Yang, S.-C. Gravitational wave emission under general parametrized metric from extreme mass ratio inspirals. arXiv 2018, arXiv:1812.04185.

14. Konoplya, R.; Rezzolla, L.; Zhidenko, A. General parametrization of axisymmetric black holes in metric theories of gravity. Phys. Rev. D 2016, 93, 064015.

15. Horava, P. Quantum gravity at a Lifshitz point. Phys. Rev. D 2009, 79, 255-215.

16. Amelino-Camelia, G. Doubly-Special Relativity: Facts, Myths and Some Key Open Issues. Symmetry 2010, 2 , 230-271.

17. Sefiedgar, A.S.; Nozari, K.; Sepangi, H.R. Modified dispersion relations in extra dimensions. Phys. Lett. B 2011, 696, 119-123.

18. Garattini, R.; Mandanici, G. Modified dispersion relations lead to a finite zero point gravitational energy. Phys. Rev. D 2011, 83, 72-12.

19. Will, C.M. Bounding the mass of the graviton using gravitational-wave observations of inspiralling compact binaries. Phys. Rev. D 1998, 57, 2061-2068.

20. Mirshekari, S.; Yunes, N.; Will, C.M. Constraining Lorentz-violating, modified dispersion relations with gravitational waves. Phys. Rev. D 2012, 85, 024041.

21. Yang, S.-C.; Han, W.-B.; Xin, S.; Zhang, C. Testing Dispersion of Gravitational Waves from Eccentric Extreme Mass-Ratio Inspirals. Int. J. Mod. Phys. D 2019, doi: 10.1142/S0218271819501669.

22. Chen, Z.; Luo, W.; Cai, Y.-F.; Saridakis, E.N. New test on General Relativity using galaxy-galaxy lensing with astronomical surveys. arXiv 2019, arXiv:1907.12225.

23. Scientific, L.I.G.O.; Abbott, B.P.; Abbott, R.; Abbott, T.D.; Acernese, F.; Ackley, K.; Adams, C.; Adams, T.; Addesso, P.; Adhikari, R.X.; et al. GW170104: Observation of a 50-Solar-Mass Binary Black Hole Coalescence at Redshift 0.2. Phys. Rev. Lett. 2017, 118, 70-17.

24. Han, W.-B.; Chen, X. Testing general relativity using binary extreme-mass-ratio inspirals. MNRAS Lett. 2019 485, L29-L33.

(C) 2019 by the authors. Licensee MDPI, Basel, Switzerland. This article is an open access article distributed under the terms and conditions of the Creative Commons Attribution (CC BY) license (http:/ / creativecommons.org/licenses/by/4.0/). 